\title{
Simplified column-switching technology for the determination of traces of anions in the presence of high concentrations of other anions
}

\author{
Yuan Huang $^{\mathrm{a}, *}$, Shi-Fen Mou ${ }^{\mathrm{a}}, \mathrm{Ke}-\mathrm{Na}$ Liu $^{\mathrm{a}}$, J.M. Rivielo ${ }^{\mathrm{b}}$ \\ ${ }^{\mathrm{a}}$ Research Center for Eco-Environmental Sciences, Academia Sinica, P.O. Box 2871, Beijing 100085, China \\ ${ }^{\mathrm{b}}$ Dionex Corporation, Sunnyvale, CA 94086, USA
}

\begin{abstract}
A binary eluent model was proposed to study the retention behavior of trace anions in the presence of high concentrations of co-eluent anions. According to this model, the effect of the matrix on the retention of trace anions can be suppressed by using a high-concentration eluent. Based on the model, a simplified 'heart-cut' column-switching technology was proposed, in which the column-switching time window can be determined directly by standard solution of the analyte. By using this technology, a detection limit of $50 \mu \mathrm{g} / \mathrm{l}$ was obtained for nitrate in the presence of $5000 \mathrm{mg} / 1 \mathrm{chloride}$ and $250 \mathrm{mg} / 1 \mathrm{sulfate}$. The effect of the matrix on the concentration efficiency of weak acid anions was studied according to the result of phosphate. The possibility of the determination of weak acid anion by the 'heart-cut' technology was also discussed. The technology has been applied for the determination of nitrate in high salinity sea water, the spike recovery is in the range of $89.2-101.3 \%$. (C) 2000 Elsevier Science B.V. All rights reserved.
\end{abstract}

Keywords: Column switching; Water analysis; Inorganic anions

\section{Introduction}

Since ion chromatography (IC) was invented in 1975 , it has become the workhorse laboratory instrument for the determination of anions [1,2]. However, for samples containing a matrix of high concentration, the determination of some anions becomes impossible owing to peak overlapping. To solve this problem, many methods were investigated. Pretreatment with $\mathrm{Ag}^{+}$or $\mathrm{Ba}^{2+}$ cartridges was used to precipitate chloride or sulfate, the most frequently encountered matrix components [3-5]. However, as the efficiency of the commercially available cartridges is inconsistent. Pretreatment cartridges are at

\footnotetext{
*Corresponding author.
}

times impractical and expensive for routine analysis [6]. Another technique called on-column matrix elimination procedure was proposed to minimize matrix effects $[7,8]$. When this procedure is employed, for different matrices, the eluent must be changed and a selective detector must be used. In recent years, 'heart-cut' column-switching technology has been employed as an general tool for the determination of trace anions in the presence of a high concentration matrix [9-11]. Since the retention time of anions varies with the concentration of the matrix, to determine the column-switching time windows, a six-step procedure is always proposed.

In recent studies, the effect of the matrix on the retention of trace anions in a carbonate-bicarbonate eluent system can be divided to three groups: the 
self-elution effect, the on column change of the eluent and on column inter-eluent neutralization $[12,13]$. Shift of the retention time of the anions is the result of these three effects. In this research work, some principles were given.

In the 'heart-cut' column-switching technology, as the determination of the column-switching time window directly depends on the retention time of the analyte [9], understanding the behavior of the trace anions in the presence of a high concentration matrix is very important. It can simplify the procedures to determine the time window and thus can shorten the time for analysis, especially when a large number of samples are analyzed.

In this paper, a binary eluent model was proposed to study the retention behavior of the trace anions in the presence of a high concentration co-eluent matrix for an hydroxide eluent system. Factors that effect the retention of the anions were studied. By controlling these factors, variation of the retention time of the anions can be reduced, thus column-switching time windows can be determined directly by the standard solutions of the analyte.

\section{Experimental}

\subsection{Chemicals}

All reagents used were of analytical reagent grade unless specified. Distilled deionized water was used throughout. All solutions were filtered through a $0.45-\mu \mathrm{m}$ membrane filter and degassed before use. Chloride and sulfate stock solutions (50 and $5 \mathrm{~g} / 1$, respectively) were prepared by dissolving $41.20 \mathrm{~g}$ of sodium chloride (Beijing Shuanghuan Chemical Reagent Factory, China) and $3.75 \mathrm{~g}$ of sodium sulfate (Beijing Chemical industrial Factory, China) in $500 \mathrm{ml}$ of deionized water. The stock solution of nitrate and phosphate $(1.000 \mathrm{~g} / 1)$ were prepared by dissolving appropriate amounts of sodium nitrate and sodium sulfate in deionized water, respectively. Standard solutions were prepared daily by serial dilution of the stock solutions prior to use. Mobile phase was $25 \mathrm{mmol} / 1 \mathrm{NaOH}$. The flow-rate is 1.0 $\mathrm{ml} / \mathrm{mm}$.

\subsection{Apparatus}

A Dionex DX-300 ion chromatograph (Sunnyvale, CA, USA) equipped with a $25-\mu l$ sample loop was employed along with a Dionex AI 450 chromatography workstation for instrument control as well as data acquisition and processing. A Dionex AG11 guard column and an AS11 separation column were used for separation. A photoelectrochemical detector in the conductivity detection mode performed detection. Suppression was achieved by a Dionex ASRS-I micromembrane suppressor in external water mode. Two high-pressure four-way valves and a concentrator column (AG11-HC, $4 \mathrm{~mm}$ ) was employed for the 'column-switching-concentration' system. The installation is illustrated in Fig. 1. A Dionex CTC-1 column and a Model DQP-1 pump were employed to eliminate the cations in the samples.

\subsection{Procedures}

This procedure consisted of matrix elimination followed by IC analysis. In the matrix elimination run, during the time windows when nitrate and phosphate eluted (3.0 3.5 min for nitrate and 4.3 $5.5 \mathrm{~min}$ for phosphate, respectively), the effluent was collected onto a concentrator column (Fig. 1A). During the remainder of the matrix elimination, and by switching a four-way valve V2, (Fig. 1B), the majority of the matrix and strongly retained anions in the samples were eluted from the column to waste. After all sample anions were eluted from the separation column $(6.0 \mathrm{~min})$, the analytical run began (Fig. 1C). The isolated nitrate and phosphate were then eluted from the concentrator column.

\section{Results and discussion}

\subsection{Theory}

Once a matrix ion enters the column, it participates in the distribution between the stationary phase and the mobile phase at the same time, acting as an eluent. Thus, trace analyte is eluted by a mixed eluent. The binary eluent model can be deducted as follows: 

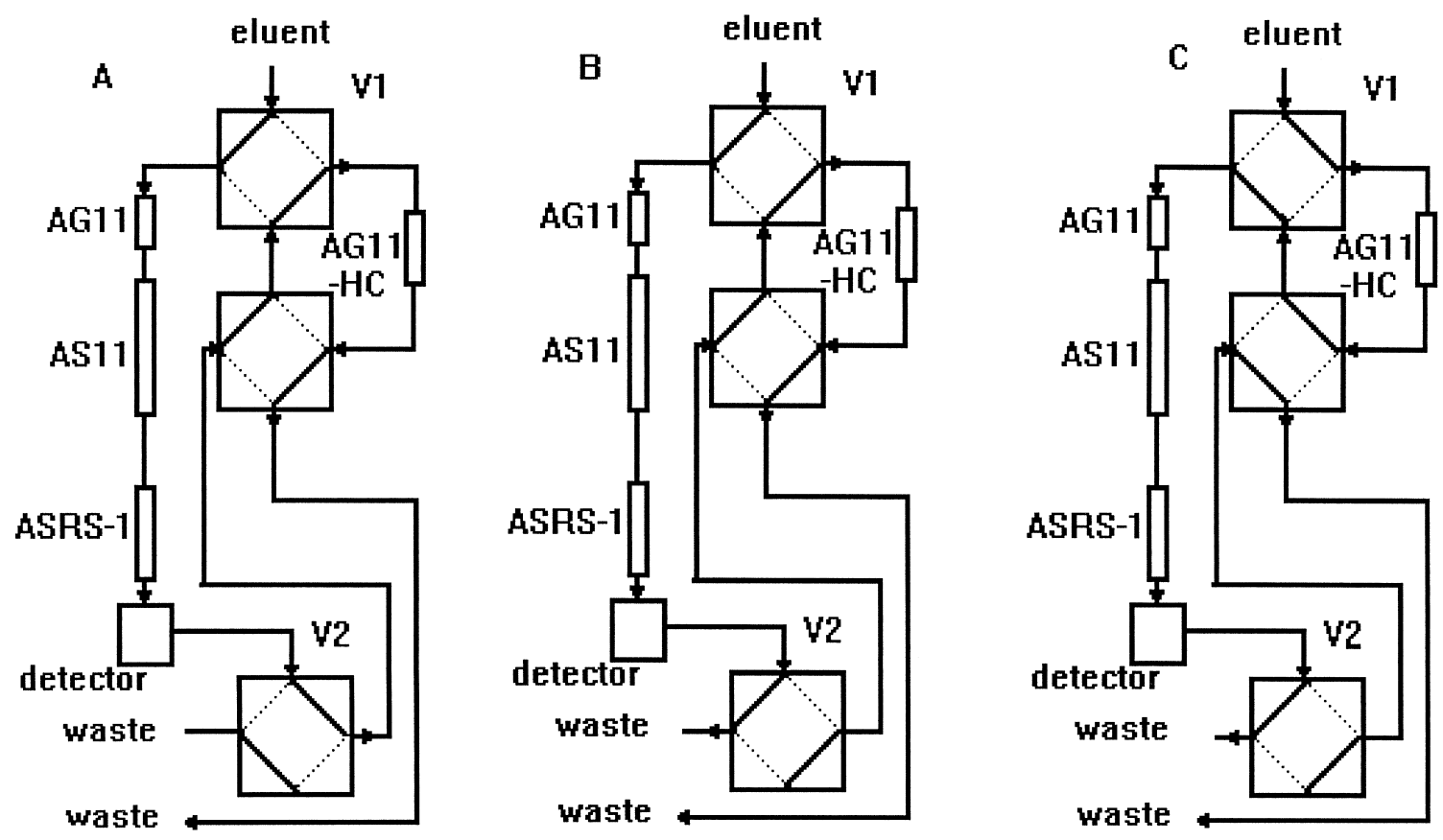

Fig. 1. System configuration. The passway is showed as the solid line. For (A), (B) and (C), see Section 2.3.

(1) Unlike the binary elution in which the stationary phase is equilibrated by the eluent, the concentration of the matrix ion in the mobile phase is a normal or Gaussian distribution function during the period in which analyte anions were eluted [14]. To simplify the derivation, the maximum concentration of the matrix, denoted as $C_{\mathrm{A}}$, is assumed as the equilibrated concentration in the mobile phase.

(2) The retention volume of the analyte is defined by the following equation [15]:

$V_{\mathrm{R}}=V_{\mathrm{M}}+K V_{\mathrm{S}}$

where $V_{\mathrm{M}}$ is the dead volume of the system, $V_{\mathrm{S}}$ is the volume of stationary phase, $K$ is the distribution coefficient of the analyte and is given by:

$K=S_{\mathrm{X}} / C_{\mathrm{X}}$

where $S_{\mathrm{X}}$ and $C_{\mathrm{X}}$ are the concentrations of analyte in the stationary and mobile phase, respectively.

In a given chromatography system, $V_{\mathrm{M}}$ and $V_{\mathrm{S}}$ are constants. Thus, the retention volume of the analyte will directly depend on the distribution coefficient $(K)$ of the analyte.

(3) The effective column capacity (denoted as $Q$ ) is expressed by the sum of adsorbed anions, including matrix anions (denoted as A) and eluent anions (denoted as B). Since the concentration of the analyte is very small. Adsorption of the analyte is neglected [16]. According to this assumption, we have:

$Q=S_{\mathrm{E}}+S_{\mathrm{A}}$.

where $S_{\mathrm{E}}$ and $S_{\mathrm{A}}$ are concentrations of the eluent and matrix anions in the stationary phase, respectively.

(4) Electroneutrality is maintained during the elution process. Since the concentration of counterions (cations) remains fixed at the mobile phase concentration (denoted as $U$ ), the sum of the concentration of matrix and eluent anions in the mobile phase must likewise equal $U$ so as to obey electroneutrality demands [17]. Also the concentration of analyte is neglected. According to this assumption, we have:

$C_{\mathrm{A}}+C_{\mathrm{E}}=U$

where $C_{\mathrm{A}}$ and $C_{\mathrm{E}}$ are the concentrations of the matrix ion and eluent ion in the mobile phase, respectively.

(5) For every pair of ionic species in the system, 
there is a constant ion-exchange equilibrium coefficient corresponding to the concentration equilibrium for the exchange reaction.

When a large amount of co-eluent ion exists in the matrix, the following equilibrium should hold for the ion-exchange resin [18]:

$S_{\mathrm{E}}=\left(K_{1} C_{\mathrm{E}}\right)\left(S_{\mathrm{X}} / C_{\mathrm{X}}\right)$

$S_{\mathrm{A}}=\left(K_{2} C_{\mathrm{A}}\right)\left(S_{\mathrm{X}} / C_{\mathrm{X}}\right)$

where $K_{1}$ and $K_{2}$ are the ion-exchange equilibrium coefficients between analyte ion and eluent ion, and between the analyte ion and the matrix ion on the ion-exchange resin. Combining Eqs. (5) and (6) with Eq. (3), one obtains:

$Q=\left(K_{1} C_{\mathrm{E}}+K_{2} C_{\mathrm{A}}\right)\left(S_{\mathrm{X}} / C_{\mathrm{X}}\right)$

Combining Eqs. (7) and (4) with Eq. (2), the value of the distribution coefficient $(K)$ of the analyte is therefore given by:

$K=\left(S_{\mathrm{X}} / C_{\mathrm{X}}\right)=Q /\left(K_{1} U-K_{1} C_{\mathrm{A}}+K_{2} C_{\mathrm{A}}\right)$

When no matrix ion exists, the value of the distribution coefficient of the analyte, denoted as $K_{0}$, is given by:

$K_{0}=\left(S_{\mathrm{X}} / C_{\mathrm{X}}\right)=Q /\left(K_{1} U\right)$

Comparing Eqs. (8) and (9), the ratio of $K_{0}$ to $K$ can be expressed as:

$$
\begin{aligned}
K_{0} / K & =\left(K_{2} C_{\mathrm{A}}-K_{1} C_{\mathrm{A}}+K_{1} U\right) /\left(K_{1} U\right) \\
& =\left[\left(K_{2} / K_{1}\right)-1\right]\left(C_{\mathrm{A}} / U\right)+1
\end{aligned}
$$

If the effect of the matrix is eliminated or effectively suppressed, the value of $K_{0} / K$ will equal or approximate 1 . Thus, we have:

$\left[\left(K_{2} / K_{1}\right)-1\right]\left(C_{\mathrm{A}} / U\right)=0$

Eq. (6) divided by Eq. (7). The value of $\left(K_{2} / K_{1}\right)$ is therefore given by:

$K_{2} / K_{1}=\left(S_{\mathrm{A}} C_{\mathrm{E}}\right) /\left(S_{\mathrm{E}} C_{\mathrm{A}}\right)$

From Eq. (12), the value of $\left(K_{2} / K_{1}\right)$ shows the preference of resin for matrix ion over the eluent ion. If this value equals to 1 , it indicates that the resin has an identical preference for these two ions.

From Eqs. (11) and (12), it shows that in order to suppress the effect of the matrix,
(1) $K_{2} / K_{1}$ equates or approximates 1 . On-column matrix elimination was proposed for the determination of trace analyte in the presence of high concentration matrices [7,8]. In this method, the matrix ion was used as a component of the eluent to overcome problems of the variable retention times and loss of chromatographic efficiency induced by a high concentration matrix. However, since the detection method employed must be selective, in most cases, conductimetric detection can not be used in this technique.

(2) $C_{\mathrm{A}} / U$ approximates to 0 . When the concentration of matrix in a sample is not too high, the value of $C_{\mathrm{A}} / U$ is small, and the peak shift of the analyte is not apparent. But with the increase of the concentration of matrix in the sample, the peak shift will be apparent. In order to suppress the peak shift, the concentration of the mobile phase $(U)$ should be as high as possible. It should be pointed out that the model was derived only when the matrix and the analyte were both monovalence anions. However, we can conclude that in other cases, with the increase of the concentration of eluent, the proportion of the matrix will decrease and thus its effect on the behavior of the analyte will also decrease.

\subsection{Separation conditions}

Undoubtedly, separation conditions for better resolution of analyte and matrix ions can result in less residual matrix introduced to the concentration column and thus can reduce the interference of the matrix more effectively. However, analysis time is also an important factor that should be considered when trying to choose the separation conditions. In addition, as discussed above, the eluent power and concentration of the eluent should also be considered. In this paper, an AS11 column was used, as this is hydroxide-selective, in which the eluent power of hydroxide is promoted and the difference in eluent power between eluent and matrix ions is reduced. From Eq. 11, we know that in this case the value of $\left(K_{2} / K_{1}-1\right)$ can be reduced.

Although peak shifts may be suppressed for the sake of the high concentration eluent, it was observed that when hydroxide of higher concentration was used (above $3 \mathrm{mmol} / \mathrm{l}$ ), background conductance increased, which means the suppressor can not 
provide enough capacity to convert the eluent to water. As a result, the residual hydroxide ions will enter the concentration column and elute some analyte from the concentration column, causing low concentration efficiency.

Sodium hydroxide at a concentration of $25 \mathrm{kmol} / \mathrm{l}$ (flow-rate $=1.0 \mathrm{ml} / \mathrm{min}$ ) was chosen as the separation condition, in which nitrate and phosphate were eluted within 3.5 and $5.5 \mathrm{~min}$, respectively. The time needed for the whole analysis is within $15 \mathrm{~min}$.

\subsection{The determination of switching time window}

To simplify the procedures for determining the time window, relatively high concentration nitrate and phosphate standards $(10 \mathrm{mg} / \mathrm{l})$ was used to determine the time window. The interval between onset and the complete elution of the standards are directly defined as the time window. There are two advantages of this method:

1. In a wide time window determined by a high concentration standard solution, the analyte of lower concentration can be introduced to the concentration column completely.

2. The shift of the analyte peak can be included in a relatively wide time window.

\subsection{Matrix elimination}

Fig. 2 illustrated that by using the column-switching technology, the interference of the matrix was effectively eliminated. Chloride at a concentration of $500 \mathrm{mg} / 1$ and $250 \mathrm{mg} / 1$ of sulfate did not interfere with the determination of nitrate and phosphate and the nitrate in seawater can be directly determined after 50 times dilution.

\subsection{Efficiency of the concentration column}

\subsubsection{Efficiency of the concentration column for anions of strong acids}

The performance of the concentration column is mainly limited by three factors: First is the efficiency of the suppressor. Because the residual hydroxide ions that are not neutralized completely in the suppressor can acted as an eluent, causing the loss of the analyte from the concentration column. The second is the time window. Since the residual

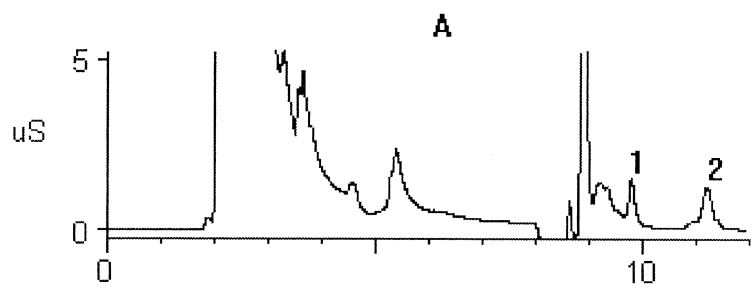

B

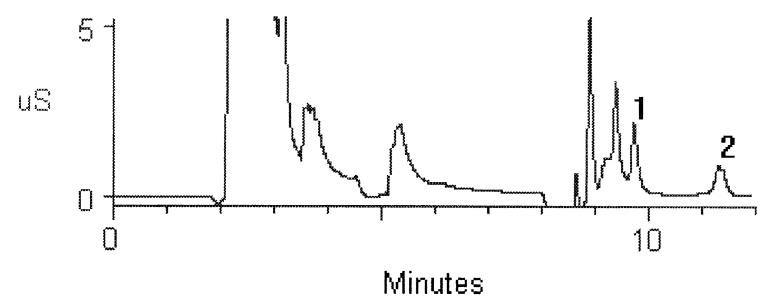

Fig. 2. Nitrate and phosphate determination on an AS11 column. Eluent: $25 \mathrm{mmol} / 1 \mathrm{NaOH}$, flow-rate $=1.0 \mathrm{ml} / \mathrm{min}$. Peaks: $1=$ $\mathrm{NO}_{\overline{3}} ; 2=\mathrm{PO}_{4}^{3-}$. (A) Mixed standard solution of $0.5 \mathrm{mg} / 1 \mathrm{NO}_{3}^{-}$ and $0.5 \mathrm{mg} / 1 \mathrm{PO}_{4}^{3-}$; matrix: $5000 \mathrm{mg} / 1 \mathrm{Cl}^{-}+250 \mathrm{mg} / 1 \mathrm{SO}_{4}^{2-}$. (B) Sea water sample after 50 times dilution.

chloride and sulfate ion can also act as an eluent. A proper time window can minimize the amount of the residual matrix and introduce the analyte to the concentration column completely. The third is the column capacity. That is, if the concentration column can provide sufficient capacity to retained the analyte and the residual matrix ions together with the residual eluent ions that are not neutralized in the suppressor.

To test the performance of the concentration column when samples of high concentration matrices were analyzed, a series of mixed standard solutions were analyzed according to the procedure described in the experimental procedure and the recoveries of the analyte retained on the concentration column were calculated. Table 1 shows the recoveries of the nitrate. It showed that, for nitrate, when concentrations were higher than $0.1 \mathrm{mg} / \mathrm{l}$, the concentration

Table 1

Recovery (\%) of nitrate

\begin{tabular}{llllrr}
\hline & \multicolumn{6}{l}{ Concentration $(\mathrm{mg} / \mathrm{l})$} \\
\cline { 2 - 6 } & 0.1 & 0.2 & 0.5 & 1.0 & 2.5 \\
\hline Recovery (\%) & 79.4 & 87.9 & 90.1 & 91.2 & 98.9 \\
\hline
\end{tabular}


column retained a high percentage of the nitrate. The high recovery of nitrate indicated that within the time window determined directly by the standard solution, a high percentage of the analyte could be introduced to the concentration column. As regards to the low recovery of $0.1 \mathrm{mg} / 1$ nitrate, we assume it is caused by the impurity of the eluent. As a result, the hydroxide is not neutralized completely in the suppressor. The residual hydroxide ion will inevitably elute some amount of the nitrate from the concentration column.

\subsubsection{Effect of the matrix on the determination of phosphate}

For phosphate, it was found that the response varied with the concentration of the matrix. To study the effect of the matrix on the response of the phosphate, a series of standard solutions consisting of different matrices and phosphate of various concentrations from 100 to $2.5 \mu \mathrm{g} / \mathrm{l}$ were analyzed according to the experimental procedure. The linear regression equations were calculated. The results are shown in Table 2. From Table 2, it was shown that the slope of the equations decreased with the increase of the concentration of the matrix. We assumed that it was due to the large concentration of the anions in the matrix. In the anion micromembrane suppressor, the suppressor exchanges all the influent cations for $\mathrm{H}^{+}$. According to assumption 4 in Section 3.1, the concentration of cations in the mobile phase remains fixed at the mobile phase concentration $(U)$, so the $\mathrm{H}^{+}$exchanged will be kept at $U$. According Eq. (4) in Section 3.1, the sum of the concentration of matrix and eluent anions in the mobile phase keeps as a constant $(U)$. When there is no matrix anions in the mobile phase, the hydrogen cations exchanged will be completely neutralized to water by the eluent anions (hydroxide anions) in the

Table 2

The linear regression equations of phosphate in different matrices

\begin{tabular}{|c|c|c|c|}
\hline \multicolumn{2}{|c|}{ Matrix (mg/1) } & \multirow[t]{2}{*}{ Linear regression equations } & \multirow{2}{*}{$\begin{array}{l}\text { Correlation } \\
\text { coefficient }\end{array}$} \\
\hline $\mathrm{Cl}^{-}$ & $\mathrm{SO}_{4}^{-}$ & & \\
\hline 0 & 0 & $y=4595146.8 x+5113.91$ & 0.996 \\
\hline 1000 & 0 & $y=581358.7 x+883$ & 0.993 \\
\hline 2000 & 100 & $y=552643.3 x+751.9$ & 0.998 \\
\hline
\end{tabular}

mobile phase. While when the matrix anion concentration in the influent suddenly increases due to the presence of large amounts of matrix anions such as chloride in the analyte sample, according to Eq. (4), the concentration of hydroxide decreases. A large amount of exchanged $\mathrm{H}^{+}$ions will not be neutralized and will co-elute with matrix anions to obey electroneutrality demands. In the case that the peak of the matrix anion completely overlaps with that of the phosphate, the large amount of hydrogen ions will cause the formation of the strong ion pair with anions of weak acids [19], such as phosphate ions. The process of proton-phosphate association may result in reducing the charge of the phosphate and then cause the loss of the phosphate from the concentration column. With the increase of the matrix anions, more $\mathrm{H}$ will be accumulated and more phosphate will form the ion pair, which can not be concentrated on the concentration column, causing the decrease of the slope.

From Table 2, it is also shown that in the same matrix, the calibration curves kept a good linear relationship. This can also be explained by the above assumption. Since the concentration of the hydronium in the suppressor is kept at the concentration of the anions in the matrix, for the same matrix, the concentration of the hydronium is the same when effluent entered the concentration column. From the association equilibration of the proton-phosphate ion pair, we know that the proportion of the ion pair directly depends on the concentration of the hydronium ions. Thus, when entering the concentration column, the proportion of the ion pair in the total phosphate is the same. In other words, for the same matrix, the proportion of the lost phosphate (ion pair) are the same regardless of the total amount of the phosphate and then the calibration curve can keep a good linear relationship.

Since for the same matrix, the good linear relationship is kept, theoretically, we can determine the concentration of the phosphate by adding a series of standard solutions and then calculate the concentration of phosphate in the samples. However, since the eluent contains a lot of phosphate ions as impurities, which will be concentrated on the concentration column and cause great bias, this method was not used for the determination of phosphate in sea samples. However, with reagents with high 
purity, it is possible to determine the phosphate in real samples.

\subsection{Accuracy and detection limit}

A series of standard solutions consisted of 500 $\mathrm{mg} / 1$ chloride, $25 \mathrm{mg} / 1$ sulfate and nitrate of various concentrations from 10 to $5 \mathrm{mg} / 1$ were analyzed according to the experimental procedure, each for three times under the same conditions. Results showed that when the concentrations were from 100 to $5 \mathrm{mg} / \mathrm{l}$, the correlation coefficient of peak area was 0.9998 . The detection limit was found to be 50 $\mathrm{mg} / \mathrm{l}$. The RSD based on the determination of 500 $\mu \mathrm{g} / 1$ nitrate $(n=7)$ is $2.5 \%$.

\section{Analysis of samples}

\subsection{Preparation of real samples}

Three seawater samples were collected form China. The samples are: No.1, from Tianjin, which is in north of China; No.2, from Lianyungang, JiangSu province in southeast of China; No.3, from Yantai, ShanDong province in north of China. All the samples were diluted 50 times with distilled deionized water. All sample solutions were injected after filtering through a $0.45-\mu \mathrm{m}$ filter. Results are listed in Table 3.

\section{Conclusion}

This simplified technology performed accurate and precise analysis for the determination of nitrate at low concentrations in seawater in a relatively short time and at low cost without pretreatment. In addi-

Table 3

Determination of nitrate in 50 times diluted seawater samples

\begin{tabular}{llllcl}
\hline Sample & Content & Added level & $n$ & Recovery $(\%)$ & RSD \\
\hline 1 & $537 \pm 5$ & 250 & 5 & 89.2 & 1.5 \\
2 & $839 \pm 3$ & 500 & 6 & 96.7 & 1.3 \\
3 & $755 \pm 6$ & 750 & 5 & 101.6 & 0.8 \\
\hline
\end{tabular}

tion, the principle of this method also showed that when column-switching technology is employed, the eluent concentration and the selectivity of the eluent ion should be considered as an important factor to suppress the shift of the analyte. Thus, we can define the proper time windows by a standard solution $[20,21]$, which will undoubtedly shorten the time required for the determination of the time windows.

\section{References}

[1] D.T. Gjerde, J.S. Fritz, Ion Chromatography, 2nd Edition, Hüthig, Mamaronek, New York, 1987.

[2] H. Small, Ion Chromatography, Plenum Press, New York, 1989, Chapter 9.

[3] F.A. Buytenhuys, J. Chromatogr. 218 (1981) 57.

[4] P. Pastore, I. Lavagnini, A. Boaretto, F. Magno, J. Chromatogr. 475 (1989) 331.

[5] R.W. Slingsby, C.A. Pohl, J. Chromatogr. A 739 (1996) 49

[6] H.S. Weinberg, H. Yamada, Anal. Chem. 70 (1998) 1.

[7] A.C.M. Brando, W.W. Buchberger, E.C.V. Butler, P.A. Fagan, P.R. Haddad, J. Chromatogr. A 706 (1995) 271.

[8] P.R. Marheni, A.R. Haddad, A.R. Mctaggart, J. Chromatogr. 546 (1991) 221.

[9] J.K. Killgore, S.R. Villasenor, J. Chromatogr. A 739 (1996) 43.

[10] S. Peldszus, P.M. Huck, S.A. Andrews, J. Chromatogr. A 793 (1998) 198.

[11] S.R. Villasenor, Anal. Chem. 63 (1991) 1362.

[12] M. Novic, B. Lecnik, V. Hudnik, B. Pihlar, J. Chromatogr. A 764 (1997) 249.

[13] M. Novic, B. Divjak, B. Pihlar, V. Hudnik, J. Chromatogr. A 739 (1996) 35.

[14] M. Vickrey Thomas, in: Liquid Chromatography Detectors, Marcel Dekker, New York, Basel, 1983, p. 12.

[15] H. Small, Ion Chromatography, Plenum Press, New York, 1989, Chapter 2.

[16] M. Maruo, N. Hirayama, T. Kuwamoto, J. Chromatogr. 481 (1989) 315.

[17] H. Small, in: Ion Chromatography, Plenum Press, New York, 1989, p. 179.

[18] H. Small, Ion Chromatography, Plenum Press, New York, 1989, Chapter 4.

[19] R.P. Singh, N.M. Abbas, S.A. Smesko, J. Chromatogr. A 733 (1996) 73.

[20] Y. Huang, S. Mou, J. Liq. Chromatogr. Rel. Technol 22 (1999) 2235.

[21] Y. Huang, S. Mou, J.M. Riviello, J. Chromatogr. A 868 (2000) 209. 